\title{
Power Efficient Strategy for Enhancing Link Capacity in Cooperative Network
}

\author{
Manisha A. Upadhyay \\ Institute of Technology, Nirma University \\ Ahmedabad, India
}

\author{
D.K. Kothari \\ Institute of Technology, Nirma University \\ Ahmedabd, India
}

\begin{abstract}
Cooperative communication has characteristics of offering spatial diversity by employing relays to improve communication efficiency significantly in wireless networks. One of the important design issues in cooperative communication is to optimize the power allocated to source and relay. The problem of allocating optimal power significantly affects the performance of the network as more power radiated by the nodes not only limit battery life but also results in increased interference in the network. On the other hand, insufficient radiated power gives rise to probability of error at the receiver. Time varying nature of the channel and mobility of nodes additionally complicate the issue of power allocation further. In this paper, Amplify \& Forward $(A F)$ and Decode \& Forward $(D F)$ cooperative systems are considered and power allocation problem with an objective of maximizing the capacity of a given link is presented. Power is allocated to source and relay depending on the channel gain between source-relay and relay-destination within the constraint of maximum total power. Capacity of the Source-RelayDestination $(S-R-D)$ link with equal power allocation and channel gain dependent power allocation have been evaluated and compared. Results show that the significant improvement in capacity of the $S-R-D$ link can be achieved with channel gain dependent power allocation compared to equal power allocation.
\end{abstract}

\section{General Terms}

Cooperative communication; outage probability; Amplify \& forward; Decode \& forward; capacity; power allocation

\section{INTRODUCTION}

Recently, cooperative communication has been seen as a promising technique to fight against fading in wireless networks. It takes advantage of the broadcast characteristic of wireless channels in which the information sent by a user can be received by neighboring nodes and then forwarded by these nodes to provide reliable communication between source and destination. The signal which conventionally was referred to as interference is now turned into useful for providing spatial diversity. This technique exhibited the performance equivalent to multiple antenna system without employing multiple antennas.

The concept of Cooperative diversity in wireless network to improve the performance is presented in [1]. The spatially distributed users create a virtual array of antennas to combat slow fading which results in increased achievable rate and capacity of wireless channels. Cooperative diversity protocol has immense impact on performance of wireless networks [2][6]. In cooperative scenario, the source of the information radiates the signal towards destination. Due to broadcast nature of wireless channel, other nodes in the vicinity are also able to receive the signal which is actually not intended for them. One or more of these intermediate nodes, process the signal and transmits it towards the destination. The destination can combine both the signals- one from source and another from relay.

There are many hurdles in the path of cooperation in wireless network. Resource allocations, security, stimulating and sustaining cooperation are the major issues which offer the challenge to the research community now a day. Out of these, resource allocation issue is undertaken in this paper.

In cooperative scenario, duplication of transmission takes place due to retransmission by one or more relays, which, in turn leads to the inefficient utilization of resources, increased traffic and increased interference. These issues can be handled by allocating the resources to the nodes based on the distance and channel condition between the pair of nodes.

\section{RELATED WORK}

The benefits of cooperation can be obtained fully by optimizing the transmission power for different Source-relay, relaydestination or source-destination pairs. The channel between each pair of nodes is time varying in nature. Therefore, appropriate power allocation strategy is the key parameter in determining better performance of cooperative wireless communication compared to traditional system [4-7]. In [4], different power allocation strategies have been developed for three-node amplify-and-forward (AF) relay schemes using either SNR or outage probability as the criteria to evaluate performance. The benefit of appropriate power allocation for AF cooperative wireless network under known channel statistics is exhibited in [6].

A power allocation scheme for maximizing the smallest of Source-Relay and Relay-Destination signal-to-noise ratios (SNRs) have been studied in [7]. Lifetime maximization of sensor node for a given probability of error in wireless sensor networks adapting cooperative protocol has been discussed in [8]. To improve the spectral efficiency of cooperative strategy and minimize the power consumption, the adaptive modulation technique is applied in [9]. In [10], power minimization problem for the entire network is presented. The symbol rate analysis and power allocation with imperfect channel state information is presented in [13].

In this paper, the movement of relay is considered and power allocation as a function of position of the relay in the network is analyzed in centrally controlled cooperative network scenario An analytical model for optimization of power in AF and DF cooperative wireless network for maximizing link capacity has been developed. Section III presents the system model and 
cooperation scheme. The analysis of power allocation scheme is presented in section IV. Section V shows that simulation results and discussion followed by conclusion in section VI

\section{SYSTEM MODEL}

\subsection{Description}

A simplified cooperative communication network is shown in Fig 1. It consists of two users transmitting to a common access point. At any instant of time, one user acts as the source while the other user serves as the relay. The coordinated communication is assumed to happen in two phases. In the first phase, the source node broadcasts its data to both the relay and the access point and in the second phase, the relay transmits the data from source to the access point.

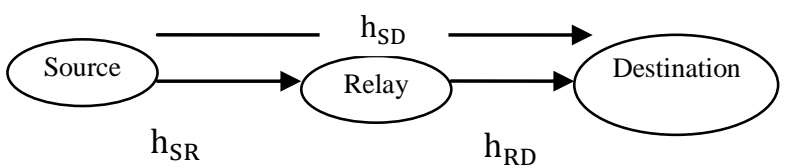

Fig 1 : Cooperative relay network of two users communicating with common access point

The two nodes interchange the roles as source node and relay node at different instants of time. Such cooperation among nodes can be enabled by employing different relay technology depending on the channel behavior, the node location, the relative mobility of nodes and the capacity of the node to withstand computational complexity.

There are many cooperative protocols like decode-and-forward (DF) [1], amplify-and-forward (AF) [1], coded cooperation (CC) [11], and compress-and-forward (CF) [12]. Out of them $\mathrm{DF}$ and $\mathrm{AF}$ are most popular relaying protocols considered by the researchers. $\mathrm{CC}$ and $\mathrm{CF}$ protocols are computationally complex and require large overheads so $\mathrm{AF}$ and $\mathrm{DF}$ protocols are mainly employed to evaluate cooperative networks. $\mathrm{AF}$ protocol is simple to implement compared to DF. In DF relaying protocol, the relay node decodes the message transmitted by the source node and forwards a newly generated message to the destination. It is assumed that the relay participates in the cooperation if and only if, it can successfully decode the message from the source. On the other hand, Amplify-and-forward (AF) relaying refers to the scheme in which the relay amplifies the analog signal received from the source and forwards it to the access point without decoding or demodulating it.

\subsection{Capacity and outage probability of a cooperative link}

The source transmits the symbol blocks to both the relay and the destination in the first phase. The received signals by relay and the destination can be given as

$$
\begin{aligned}
& y_{R}=\left|h_{S R}\right|^{2} \sqrt{P_{S}} x_{S}+w_{R} \\
y_{D(\text { phasel })}= & \left|h_{S D}\right|^{2} \sqrt{P_{S}} x_{S}+w_{R}
\end{aligned}
$$

Where, $x_{S}$ is the transmitted symbol by the source and $y_{R}$ and $y_{D}$ is the revived symbol at relay and destination respectively, $\left|h_{S R}\right|^{2}$ is the channel gain for source-relay link, $\left|h_{R D}\right|^{2}$ is the channel gain for relay-destination link and $\left|h_{S D}\right|^{2}$ is the channel gain of source-destination link. For the sack of simplicity, we consider the path loss model in which channel gain depend on the distance between the nodes S-R, R-D and S-D. The distance between S-R, R-D and S-D pairs are $d_{S R}, d_{R D}$ and $d_{S D}$, respectively. $P_{S}$ and $P_{R}$ are the source power and relay power, respectively. $w_{R} \sim \operatorname{CN}\left(0, \sigma_{\mathrm{SR}}^{2}\right)$, and $w_{D} \sim \operatorname{CN}\left(0, \sigma_{\mathrm{RD}}^{2}\right)$, are AWGN at the relay and destination with variances $\sigma_{\mathrm{SR}}^{2}$ and $\sigma_{\mathrm{RD}}^{2}$.

In the second phase, the relay sends the signal to destination received by it in the previous phase.

$$
y_{D(\text { phaselI })}=\left|h_{R D}\right|^{2} \sqrt{P_{R}} y_{R}+w_{D}
$$

The Destination combines both the replicas of the signal received by it during phase I \& II using Maximum ratio combining (MRC) technique.

In case of DF protocol, the received SNR at Destination can be given as

$$
S N R_{S R D}^{D F}=\frac{\left|h_{S D}\right|^{2} P_{S}}{\sigma_{\mathrm{R}}^{2}}+\frac{\left|h_{R D}\right|^{2} P_{R}}{\sigma_{\mathrm{D}}^{2}}=\gamma_{S D}+\gamma_{R D}
$$

where $\gamma_{S D}$ and $\gamma_{R D}$ are signal to noise ratio (SNR) for S-D and R-D links, respectively. Achievable data rate at the destination is limited by the minimum SNR of link between source and relay and combined SNR. In DF, the cooperation is meaningful if and only if the relay is able to decode the message successfully. To enable relay to do so, the data from the source to relay must be sent at the lower rate than its capacity. Capacity of the link will be limited by the minimum of the SNR of the SR and SD links and can be estimated by equation (5)

$$
C_{S R D}^{D F}=\frac{1}{2} \min \left[\log _{2}\left(1+\gamma_{S R}\right), \log _{2}\left(1+\gamma_{S D}+\gamma_{R D}\right)\right]
$$

where $\gamma_{S R}$ is the signal-to-noise ratio of S-R link. The factor half in the equation (5) is due to duplication of transmission. To make fair comparison with capacity without cooperation, it is necessary to use factor of $1 / 2$ in equation (5).

Outage Probability is the probability of $C_{S R D}^{D F}$ to be less than the desired data rate $\mathrm{R}$ to satisfy certain quality of service requirements.

$$
\begin{aligned}
& p_{\text {ouatge } D F}=P_{r}\left\{\frac{1}{2} \min \left[\log _{2}\left(1+\gamma_{S R}\right), \log _{2}\left(1+\gamma_{S D}+\gamma_{R D}\right)\right]\right. \\
& <\mathrm{R}\} \\
& =P_{r}\left\{\min \left[\log _{2}\left(1+\gamma_{S R}\right), \log _{2}\left(1+\gamma_{S D}+\gamma_{R D}\right)\right]<2 \mathrm{R}\right\}
\end{aligned}
$$

In case of Amplify \& Forward, a relay receives the signal and re-transmits it by amplifying it. The gain of the amplifier depends on the channel gain. At destination, both the signals are combined using MRC. Combined SNR can be given as

$$
\begin{aligned}
S N R_{S R D}^{A F}= & \frac{\left|h_{S D}\right|^{2} \mathrm{P}_{\mathrm{S}}}{\sigma_{\mathrm{D}}^{2}}+\frac{\frac{\left|h_{S R}\right|^{2} \mathrm{P}_{\mathrm{S}}}{\sigma_{\mathrm{R}}^{2}} \cdot \frac{\left|h_{R D}\right|^{2} \mathrm{P}_{\mathrm{R}}}{\sigma_{\mathrm{D}}^{2}}}{\frac{\left|h_{S R}\right|^{2} \mathrm{P}_{\mathrm{S}}}{\sigma_{\mathrm{R}}^{2}}+\frac{\left|h_{R D}\right|^{2} \mathrm{P}_{\mathrm{R}}}{\sigma_{\mathrm{D}}^{2}}+1} \\
=\gamma_{S D}+\frac{\gamma_{S R} \cdot \gamma_{R D}}{\gamma_{S R}+\gamma_{R D}+1} &
\end{aligned}
$$

Maximum capacity for AF, then can be given as

$$
C_{S R D}^{A F}=\frac{1}{2}\left[\log _{2}\left(1+\gamma_{S D}+\frac{\gamma_{S R} \cdot \gamma_{R D}}{\gamma_{S R}+\gamma_{R D}+1}\right)\right]
$$

Outage probability for AF protocol can be given as

$$
p_{\text {out }, A F}=P_{r}\left\{\frac{1}{2}\left[\log _{2}\left(1+\gamma_{S D}+\frac{\gamma_{S R} \cdot \gamma_{R D}}{\gamma_{S R}+\gamma_{R D}+1}\right)\right]<R\right\}
$$




$$
=P_{r}\left\{\left(\gamma_{S D}+\frac{\gamma_{S R} \cdot \gamma_{R D}}{\gamma_{S R}+\gamma_{R D}+1}\right)<2^{2 R}-1\right\}
$$

It can be seen from equations (5) \& (6) that capacity of the link depends of the signal to noise ratio and channel coefficients of the path between each pair of nodes. In place of allocating fixed powers to sources and the relay, we propose to allocate power to source and relay nodes depending on the channel coefficient so as to maximize the capacity and hence minimize the outage probability. Total power to be allocated to all the users is constrained to maximum power available in case of fixed power allocation so as to make comparison with non-cooperative transmission. Following section presents formulation of power optimization problem and its analytical solution.

\section{ANALYSIS OF OPTIMAL POWER ALLOCATION FOR MAXIMIZING CAPACITY}

In the wireless scenario, the channel is time varying in nature. As a result, the fixed equal power allocation to the nodes results in inefficient utilization of the resource, reduced capacity and may tend to increase outage probability. Power can be allocated to the nodes in optimized manner by exploiting instantaneous Channel State Information (CSI) available at the transmitter.

\subsection{Optimization problem formulation for DF protocol}

Optimal power allocation problem with an objective of maximizing the capacity for DF protocol is formulated as shown below.

\section{Objective Function 1}

$\max _{P_{S}, P_{R}} \quad \frac{1}{2} \min \left\{\log _{2}\left(1+\frac{\left|h_{S R}\right|^{2} P_{S}}{\sigma_{\mathrm{R}}^{2}}\right), \quad \log _{2}\left(1+\frac{\left|h_{S D}\right|^{2} P_{S}}{\sigma_{\mathrm{D}}^{2}}+\right.\right.$ hRD2PRøD2

Subject to:

$$
\begin{aligned}
& \text { a) } P_{S}, P_{R}>0 \\
& \text { b) } P_{S}+P_{R} \leq 2 P_{\max } \quad \ldots . P_{\max }=P_{s}=P_{R}
\end{aligned}
$$

Constraint (a) ensures some minimum power is to be allocated to each node. Constraint (b) indicates that total power available for allocation is limited to twice the maximum power of source and relay.

It can be seen that the capacity is the function of power so maximum capacity can be achieved when power $2 P_{\max }$ entirely is distributed between the source and the relay.

Relay power can, then, be given as

$$
\mathrm{P}_{\mathrm{R}}=2 \mathrm{P}_{\max }-\mathrm{P}_{\mathrm{S}}
$$

Putting (10) in the objective function 1, we get

$$
\begin{aligned}
\max _{S} \frac{1}{2} \min \left\{\log _{2}\left(1+\frac{\left|h_{S R}\right|^{2} P_{S}}{\sigma_{\mathrm{R}}^{2}}\right), \log _{2}(1\right. \\
\left.\left.+P_{S}\left(\frac{\left|h_{S D}\right|^{2}-\left|h_{R D}\right|^{2}}{\sigma_{\mathrm{D}}^{2}}\right)+\frac{\left|h_{R D}\right|^{2} 2 P_{\max }}{\sigma_{\mathrm{D}}^{2}}\right)\right\}
\end{aligned}
$$

The effect of channel condition between the node pairs S-R, R$\mathrm{D}$ and S-D greatly affects the power allocation. When $\left|h_{S D}\right|^{2} \geq$ $\left|h_{R D}\right|^{2}$, maximum capacity can be obtained by setting $2 P_{\max }=$ $P_{S}$ i.e. when the direct path between the source and the destination is good, cooperation is not required at all. In case of $\left|h_{S D}\right|^{2}<\left|h_{R D}\right|^{2}$, maximum capacity can be obtained when

$$
\begin{aligned}
& \log _{2}\left(1+\frac{\left|h_{S R}\right|^{2} P_{S}}{\sigma_{\mathrm{R}}^{2}}\right)=\log _{2}\left(1+P_{S}\left(\frac{\left|h_{S D}\right|^{2}-\left|h_{R D}\right|^{2}}{\sigma_{\mathrm{D}}^{2}}\right)+\right. \\
& \text { hRD22PmaxбD2 (11) }
\end{aligned}
$$

Solving (10), the optimized power allocation can be obtained as

$P_{S}=2 P_{\max }\left[\frac{\left|h_{R D}\right|^{2}}{\sigma_{\mathrm{D}}^{2}} / \frac{\left|h_{S R}\right|^{2}}{\sigma_{\mathrm{R}}^{2}}+\frac{\left(\left|h_{R D}\right|^{2}-\left|h_{S D}\right|^{2}\right)}{\sigma_{\mathrm{D}}^{2}}\right]$
$P_{R}=2 P_{\max }\left[\frac{\left|h_{S R}\right|^{2}}{\sigma_{\mathrm{R}}^{2}}-\frac{\left|h_{S D}\right|^{2}}{\sigma_{\mathrm{D}}^{2}} / \frac{\left|h_{S R}\right|^{2}}{\sigma_{\mathrm{R}}^{2}}+\frac{\left(\left|h_{R D}\right|^{2}-\left|h_{S D}\right|^{2}\right)}{\sigma_{\mathrm{D}}}\right]$

$P_{R}=2 P_{\max }\left[\frac{\left|h_{S R}\right|^{2}}{\sigma_{\mathrm{R}}^{2}}-\frac{\left|h_{S D}\right|^{2}}{\sigma_{\mathrm{D}}^{2}} / \frac{\left|h_{S R}\right|^{2}}{\sigma_{\mathrm{R}}^{2}}+\frac{\left(\left|h_{R D}\right|^{2}-\left|h_{S D}\right|^{2}\right)}{\sigma_{\mathrm{D}}^{2}}\right]$

\subsection{Optimization problem formulation for AF protocol}

In case of AF protocol, the optimization problem can be formulated as

\section{Objective Function 2}

$$
\begin{aligned}
& \max _{P_{S}, P_{R}} \quad \frac{1}{2}\left[\operatorname { l o g } _ { 2 } \left(1+\frac{\left|h_{S D}\right|^{2} P_{S}}{\sigma_{\mathrm{D}}^{2}}\right.\right. \\
& \left.\left.+\frac{\frac{\left|h_{S R}\right|^{2} P_{S}}{\sigma_{\mathrm{R}}^{2}} \frac{\left|h_{R D}\right|^{2} P_{R}}{\sigma_{\mathrm{D}}^{2}}}{\frac{\left|h_{S R}\right|^{2} P_{S}}{\sigma_{\mathrm{R}}^{2}}+\frac{\left|h_{R D}\right|^{2} P_{R}}{\sigma_{\mathrm{D}}^{2}}+1}\right)\right]
\end{aligned}
$$

Subject to:

$$
\begin{aligned}
& \text { c) } P_{S} P_{R}>0 \\
& \text { d) } P_{S}+P_{R} \leq 2 P_{\max } \quad \ldots . P_{\max }=P_{s}=P_{R}
\end{aligned}
$$

Constraint (c) ensures some minimum power is to be allocated to each node. Constraint (d) indicates that total power available for allocation is limited to twice the maximum power of source and relay.

Putting (10) and assuming $\sigma_{\mathrm{D}}^{2}=\sigma_{\mathrm{R}}^{2}=1$, we get

$$
\begin{aligned}
\max _{P_{S}, P_{R}} & \frac{1}{2}\left[\operatorname { l o g } _ { 2 } \left(1+\left|h_{S D}\right|^{2} P_{S}\right.\right. \\
& \left.\left.+\frac{\left|h_{S R}\right|^{2} P_{S} \cdot\left|h_{R D}\right|^{2}\left(2 P_{\max }-P_{S}\right)}{\left|h_{S R}\right|^{2} P_{S}+\left|h_{R D}\right|^{2}\left(2 P_{\max }-P_{S}\right)+1}\right)\right]
\end{aligned}
$$

Above problem can be solved by taking first derivative and equating it to zero, the optimum power $P_{S}$ and $P_{R}$ can be obtained.

$$
\begin{aligned}
& \frac{d}{d P_{S}}\left[\frac { 1 } { 2 } \left[\operatorname { l o g } _ { 2 } \left(1+\left|h_{S D}\right|^{2} P_{S}\right.\right.\right. \\
& \left.\left.\left.+\frac{\left|h_{S R}\right|^{2} P_{S} \cdot\left|h_{R D}\right|^{2}\left(2 P_{\max }-P_{S}\right)}{\left|h_{S R}\right|^{2} P_{S}+\left|h_{R D}\right|^{2}\left(2 P_{\max }-P_{S}\right)+1}\right)\right]\right]=0
\end{aligned}
$$

Solving,

$$
\begin{gathered}
P_{S}=\frac{2 P_{\max }\left(\left|h_{S R}\right|^{2}\left|h_{R D}\right|^{2}+\left|h_{S D}\right|^{2}\left|h_{S R}\right|^{2}\right)+\left|h_{S D}\right|^{2} / 2 P_{\max }}{\Psi+\sqrt{\frac{1+2 P_{\max }\left|h_{S R}\right|^{2}}{1+2 P_{\max }\left|h_{R D}\right|^{2}}\left|h_{S R}\right|^{2}\left|h_{R D}\right|^{2}} \Psi} \\
P_{R}=\frac{2 P_{\max }\left(\left|h_{S R}\right|^{2}\left|h_{R D}\right|^{2}-\left|h_{S D}\right|^{2}\left|h_{S R}\right|^{2}\right)-\left|h_{S D}\right|^{2} / 2 P_{\max }}{\Psi+\sqrt{\frac{1+2 P_{\max }\left|h_{S R}\right|^{2}}{1+2 P_{\max }\left|h_{R D}\right|^{2}}\left|h_{S R}\right|^{2}\left|h_{R D}\right|^{2}} \Psi}
\end{gathered}
$$


Where $\Psi=\left|h_{S R}\right|^{2}\left|h_{R D}\right|^{2}+\left|h_{S D}\right|^{2}\left|h_{R D}\right|^{2}-\left|h_{S R}\right|^{2}\left|h_{S D}\right|^{2}$.

In special case, when relay is at half the way between the source and the destination. $\left|h_{S R}\right|^{2}=\left|h_{R D}\right|^{2}=\left|h_{S D}\right|^{2} / 2$. By putting this condition in equation (14) and (15), optimized power can be computed as

$$
\begin{gathered}
P_{S}=\frac{6 P_{\max }\left|h_{S R}\right|^{2}+2}{\left|h_{S R}\right|^{4}\left(\left|h_{S R}\right|^{2}+1\right)} \\
P_{R}=\frac{2 P_{\max }\left|h_{S R}\right|^{2}-2}{\left|h_{S R}\right|^{4}\left(\left|h_{S R}\right|^{2}+1\right)}
\end{gathered}
$$

Equations (16) and (17) show that the optimized power of source and relay depend on the channel gain, considering $P_{\max }$, which in turn depend on the distance between the node pairs in our case.

\section{SIMULATION RESULT AND DISCUSSION}

In this section, the simulation results of capacity and outage probability Amplify \& forward and Decode \& forward protocols in cooperative communication systems are presented.

\subsection{Simulation environment}

The relay is assumed to move from source towards destination. The distance between Source and relay increases while the distance between relay and destination reduces as the relay moves from source to destination. The channels between the nodes are modeled with distance dependent path loss model with path loss exponent 4 . Other channel effects are neglected for the sack of simplicity. Centrally control wireless network is assumed here in which the destination is assumed to have channel state information of all the users in the network. The destination calculates the power of source and relay which can result in maximum capacity of the link for the current position of the relay. The same information is sent to the source and the relay through feedback channel.

\subsection{Power allocation algorithm}

Step:1 Destination collects the information of channel gains $\left|h_{S R}\right|^{2},\left|h_{R D}\right|^{2}$ and $\left|h_{S D}\right|^{2}$.

Step:2 Destination checks for $\left|\mathrm{h}_{\mathrm{SD}}\right|^{2}<\left|\mathrm{h}_{\mathrm{SR}}\right|^{2}$ and $\left|\mathrm{h}_{\mathrm{RD}}\right|^{2}$.If $\mathrm{S}-$ $\mathrm{D}$ channel is poor compared to S-R and R-D channels, then cooperative protocol takes steps 3 and 4 to improve performance.

If $\left|h_{\mathrm{SD}}\right|^{2}>\left|\mathrm{h}_{\mathrm{SR}}\right|^{2}$ and $\left|\mathrm{h}_{\mathrm{RD}}\right|^{2}$, the direct channel (S-D) is better compared to $\mathrm{S}-\mathrm{R}$ and $\mathrm{R}-\mathrm{D}$ links so no cooperation is required.

Step:3 Destination calculates power of Source $P_{S}$ and Relay $\mathrm{P}_{\mathrm{R}}$ which can result in maximum capacity for the given value of channel gains from equations (14) and (15) and send it to Source and relay through feedback channel.

Step:4 Source and relay transmit with the optimum power as calculated by the destination

Step: 5 Go back to step: 1

\subsection{Outage probability with and without cooperation}

It can be seen from equations (6) and (9) that outage probability reduces when capacity of the channel becomes higher. As cooperation results in increased capacity, the probability of outage can be reduced by employing cooperation. The outage probability with respect to position of the relay is depicted in Fig [2]. It compares probability of outage for direct transmission between the source and the destination with and without cooperation from the relay for AF protocol. The relay moves up to 100 meters from the source towards destination with the steps of 10 meters. In case of no cooperation, the value of outage probability is significantly higher. For cooperative link, when relay starts moving from source, outage probability starts reducing and becomes the lowest when the relay is at the centre position. It shows that the probability of outage can be reduced by employing cooperation.

\subsection{Capacity of AF and DF protocol}

Fig [3] shows the capacity of AF and DF with the moving relay. The relay moves $100 \mathrm{~m}$ distance from the source to destination. When the relay is at half the way between the source and the destination, maximum capacity can be achieved. It also shows the comparison of capacity with $\mathrm{AF}$ and $\mathrm{DF}$ protocols. DF protocol results in higher capacity compared to $\mathrm{AF}$ when relay is not too close to either source or destination In DF protocol, the performance of the cooperative link is limited by the worse of the two S-R and R-D link as shown by equations (5) and (6). As a result, when relay is near to either Source or Destination, AF performs better than DF whereas for relay positions between 35 meter to 60 meter, DF protocol results in higher capacity. At the centre position of the relay, capacity of AF is $4.43 \mathrm{bits} / \mathrm{sec} / \mathrm{Hz}$ and that of DF is $4.88 \mathrm{bits} / \mathrm{sec} / \mathrm{Hz}$.

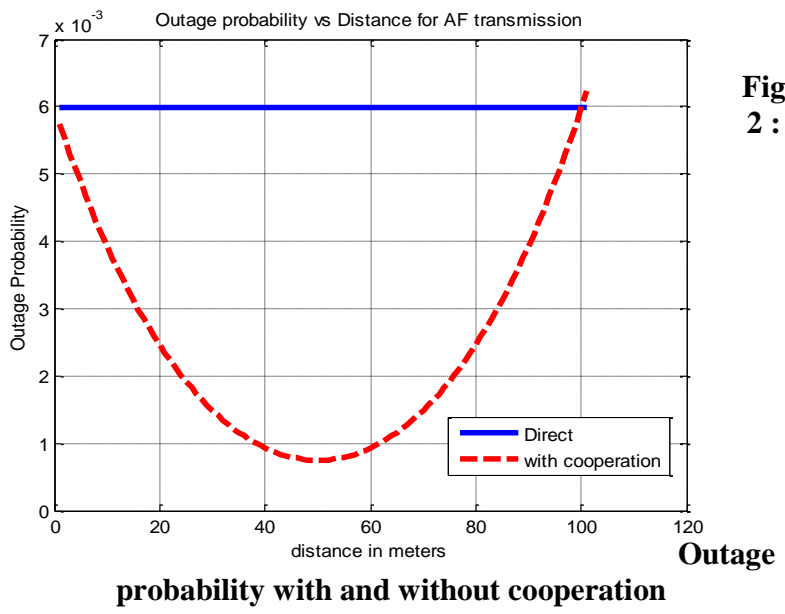




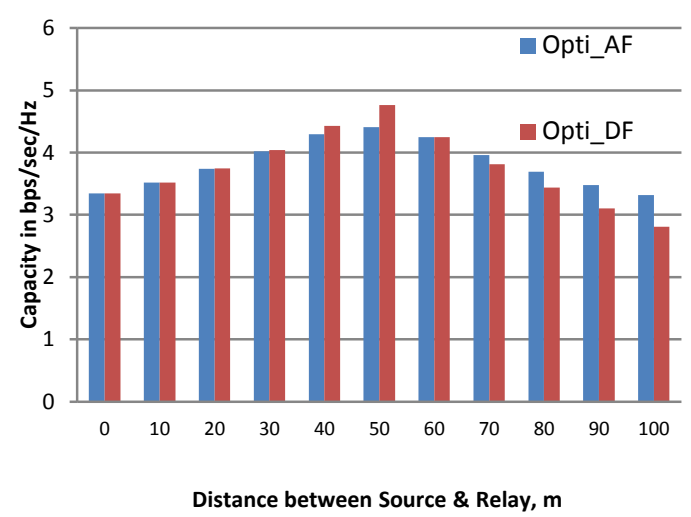

Fig 3 : Capacity of source destination link with the help of relay by allocating optimum power to source and relay

In $\mathrm{AF}$, the relay retransmits the amplified version of the message received by the source without correcting the errors. As a result the accumulation of error can be avoided in case of DF. It is apparent from fig.[3] that capacity achieved by DF is more compared to $\mathrm{AF}$ assuming error correction is perfect in DF. But as the relay movers away from the centre position, the capacity of DF drops more quickly compared to AF as in DF, the capacity is determined by the worse of the two channels and in $\mathrm{AF}$, it depends on the average of the two channels.

\subsection{Optimized power allocation to Source and Relay}

In Fig [4], the power allocated to source and relay is compared for equal allocation and optimized allocation. Total power of $2 \mathrm{~W}$ is equally divided between the source and relay so each one will transmit $1 \mathrm{~W}$ power in case of equal allocation irrespective of the position of relay. While in case of optimized allocation, power allocated to the source and relay depends on the position of the relay. When the relay is closed to the source, the channel between the source and the relay is good compared to that of relay and the destination. So source is assigned less power and relay is assigned more power to transmit which in turn results in maximum capacity of the SRD link.

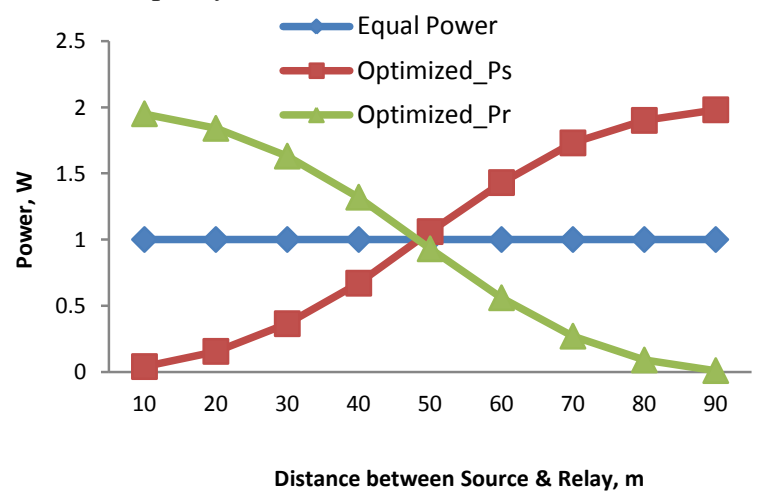

Fig 4 : Power allocated to source \& relay in case of optimized allocation compared to equal power allocation considering that the relay moves from source to destination

\subsection{Comparison of capacity with equal and optimized power}

The increase in capacity with optimized power allocation for AF protocol and DF protocol employed at relay is depicted in Fig.[5] and Fig.[6] respectively.

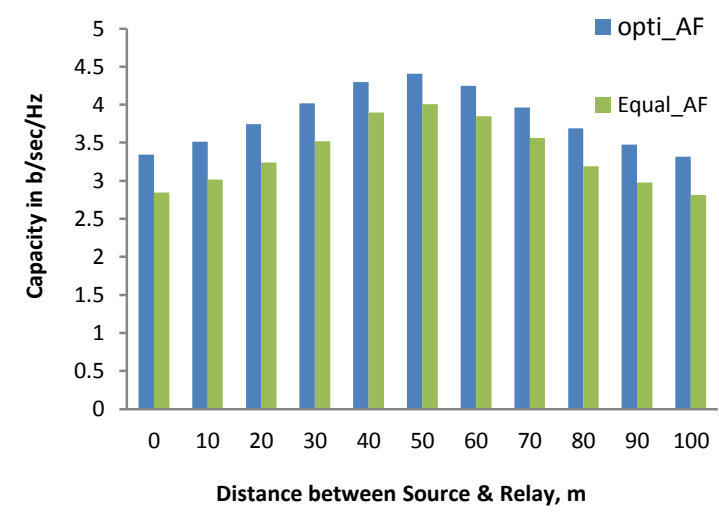

Fig 5 : Capacity with equal power allocation and with optimized allocation for AF protocol at relay

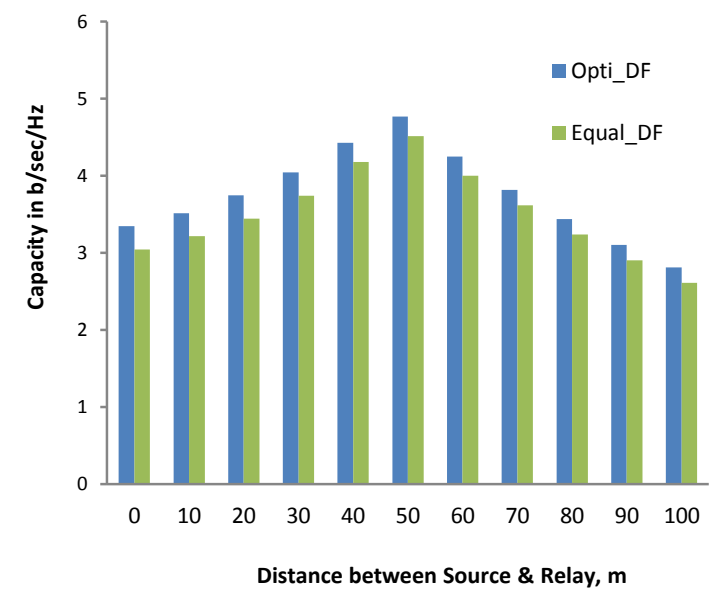

Fig 6 : Capacity with equal power allocation and with optimized allocation for DF protocol at Relay

Fig [5] \& [6] show the significant increase in capacity obtained as a result of optimized power allocation. The increase in capacity in case of $\mathrm{AF}$ ranges from $0.4 \mathrm{bits} / \mathrm{sec} / \mathrm{Hz}$ to 0.54 bits $/ \mathrm{sec} / \mathrm{Hz}$ with an average of $0.48 \mathrm{bits} / \mathrm{sec} / \mathrm{Hz}$. In case of DF, the increase in capacity ranges from $0.3 \mathrm{bits} / \mathrm{sec} / \mathrm{Hz}$ to 0.38 $\mathrm{bits} / \mathrm{sec} / \mathrm{Hz}$ with an average increase of $0.34 \mathrm{bits} / \mathrm{sec} / \mathrm{Hz}$. Here, movement of relay in one direction is considered. However, this concept can be further employed to the movement of relay in any direction. Further, this technique can be applied to multiuser network to get more sum total capacity of a network by optimal allocation of the power.

\section{CONCLUSION}

In this paper, power allocation strategy to enhance the capacity of cooperative link has been presented. The relay moving in straight line between the source and the destination has been considered here. In place equal power being allocated to source and the relay, we have proposed to allocate the power allocation 
to both of them on the basis of channel gains of S-R and R-D links. Channel gain based power allocation has shown significant enhancement in capacity of $S-R-D$ cooperative link. Capacity of $S-R-D$ link is, on average, increased by 0.48 $\mathrm{bits} / \mathrm{sec} / \mathrm{Hz}$ in case of $\mathrm{AF}$ protocol and $0.34 \mathrm{bits} / \mathrm{sec} / \mathrm{Hz}$ for DF protocol. Increase in the link capacity is also attributed by reduction in the probability of outage. The outage probability becomes the minimum when the relay is exactly half the way between the source and the destination. Results clearly show that the benefits of cooperation can be achieved by allocating the resources to the participating nodes in optimized way in the network. For the sake of simplicity, three- node network is considered here. However, the same concept can be further extended for the multi-user network to obtain significant increase in link capacity.

\section{REFERENCES}

[1] J. N. Laneman, D. N. C. Tse, and G. W. Wornell, Cooperative diversity in wireless networks: Efficient protocols and outage behavior," IEEE TransInform. Theory, vol. 50, pp. 3062-3080, Dec. 2004.

[2] L. Le and E. Hossain, "Multihop cellular networks: Potential gains, research challenges, and a resource allocation framework," IEEE Commun. Mag., vol. 45, pp. 66-73, Sept. 2007.

[3] Y. Li, B. Vucetic, Z. Zhou, and M. Dohler, "Distributed adaptive power allocation for wireless relay networks," IEEE Trans. Wireless Commun.,vol. 6, pp. 948-958, Mar. 2007.

[4] X. Deng, and A. M. Haimovich, "Power allocation for cooperative relaying in wireless networks," IEEE Commun. Letters, vol. 9, pp. 994-996, Nov. 2005.

[5] Y. Liang, and V. Veeravalli, "Gaussian orthogonal relay channel: optimal resource allocation and capacity," IEEE Trans. Inform. Theory, vol. 51, no. 9, pp. 3284-3289, Sept. 2005.
[6] Y. Zhao, R.S. Adve, and T.J. Lim, "Improving amplifyand-forward relay networks: optimal power allocation versus selection," IEEE Trans. Wireless Commun., vol. 6, no. 8, pp. 3114-3123, Aug. 2007.

[7] V. Havary-Nassab, S. Shahbazpanahi, and A. Grami, "Optimal distributed beamforming for two-way relay networks," IEEE Trans. Signal Process., vol. 58, no. 3, pp. 1238-1250, Mar. 2010.

[8] T. Himsoon, W.P. Siriwongpairat, Z. Han, and K.J.R Liu,"Lifetime Maximization via Cooperative Nodes and Relay Deployment in Wireless Networks", IEEE Journal of Selected Areas in Communications, vol 25, no 2, pp.306-317, Feb 2007

[9] E. Yazdian, M.R. Pakravan, “Adaptive Modulation Technique for Cooperative Diversity in Wireless Fading Channel," PIMRC, Sep 2006.

[10] V. Mahinthan, L. Cai, J. W. Mark and X. Shen, "Maximizing Cooperative Diversity Energy Gain for Wireless Networks," IEEE Trans. Wireless Commun., vol. 6, no. 7, pp.2530-2539, Jul. 2007.

[11] Hunter, T., Nosratinia, A.: Cooperative diversity through coding. In: Proceedings on the IEEE International Symposium on Information Theory (ISIT) pp. 220 (2002)

[12] Hu, R., Li, J.: Practical compress-and-forward in user cooperation: Wyner-Ziv cooperation. In: Proceedings on the IEEE International Symposium on Information Theory (ISIT) pp. 489-493 (2006)

[13] Mehdi Basiri Abarghouei, Ali M. Doost Hoseini," Cooperative communication with imperfect channel information:Performance analysis and optimum power allocation", Elsevier Journal of Physical Communication, Volume 4, Issue 3, September 2011, Pages 144-155 